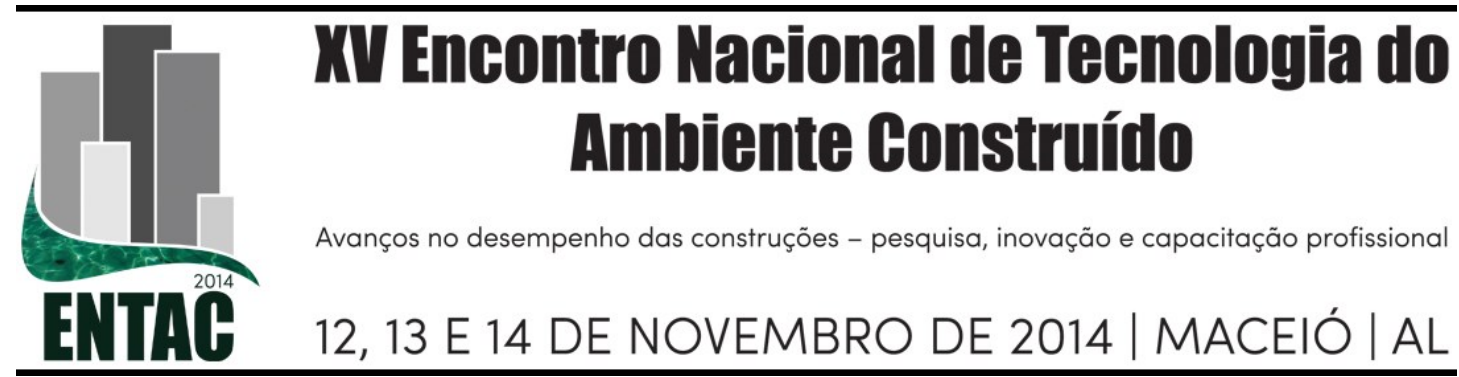

\title{
SIMULAÇÃO FÍSICA EM HELIODON COMPUTADORIZADO NO ENSINO DA CONCEPÇÃO ARQUITETÔNICA BIOCLIMÁTICA
}

\section{ZAMBRANO, Letícia M. A. (1); CASTRO, Eduardo B. P. (2), GOMES, Francisco (3), MARQUES, Aline C. (4), BASTOS, Pedro K. X. (5), MACHADO, Ernani (6), FONTES, Pâmela (7), CABRAL, Monique C. (8), SANTOS, Caroline V.M. (9), CARVALHO, Diego A. (10), CYRILLO, Yasmin (11)}

(1) Universidade Federal de Juiz de Fora, tel. 21023403 r. 217, e-mail: leticia.zambrano@ufjf.edu.br (2) Universidade Federal de Juiz de Fora, e-mail: eduardo.castro@ufff.edu.br (3) Universidade Federal de Juiz de Fora, e-mail: chico.gomes@ufjf.edu.br (4) INSA Toulouse, e-mail: acmaquetes@yahoo.com.br

(5) Universidade Federal de Juiz de Fora, e-mail: pedrokop@terra.com.br (6) Universidade Federal de Juiz de Fora, e-mail: ernani.machado@ufjf.edu.br (7) Universidade Federal de Juiz de Fora, e-mail: pamelafontes@hotmail.com (8) Universidade Federal de Juiz de Fora, e-mail: moniquecastrocabral@gmail.com (9) Universidade Federal de Juiz de Fora, e-mail: carolvazdemello@gmail.com (10) Universidade Federal de Juiz de Fora, e-mail: diego.carvalho@engenharia.ufff.br (11) Universidade Federal de Juiz de Fora, e-mail: yasmin.cyrillo@engenharia.ufjf.br.

\begin{abstract}
RESUMO
O ensino da concepção arquitetônica bioclimática envolve, entre diversos aspectos, o conhecimento da geometria solar, um dos elementos fundamentais para o desempenho ambiental da edificação. Atualmente, em função das facilidades promovidas pelos programas de simulação computacional, os estudantes de arquitetura realizam estudos de insolação utilizando modelos numéricos, o que os afasta do conhecimento efetivo da geometria solar. Este trabalho apresenta o equipamento de simulação física, comandado por computador, que foi desenvolvido para o Laboratório de Conforto Ambiental e Sustentabilidade - ECOS, e seu potencial de aplicação no ensino. Trata-se do resultado de uma pesquisa aplicada, para desenvolvimento de uma versão inovadora - com comando numérico - de um dispositivo clássico de simulação física - o heliodon. O objetivo do artigo é descrever os materiais e métodos utilizados no desenvolvimento do equipamento e apresentar o teste piloto realizado para validação do mesmo, baseado em análises comparativas entre simulação física e computacional. A metodologia da pesquisa baseou-se no estudo bibliográfico outros equipamentos similares, incorporando como inovação o comando numérico para movimentação. Os resultados do teste piloto demonstraram a adequação do equipamento em relação ao funcionamento geral e ângulos solares, entretanto verifica-se a necessidade de ajuste do projetor de iluminação em função de deformação dos ângulos de incidência da luz. Os testes demonstraram que utilizando o heliodon, conjuntamente com simulações computacionais, os estudantes dispõem de um instrumental mais completo para compreender o fenômeno físico da trajetória aparente do sol. Este conhecimento permite melhorar o domínio da orientação da edificação e disposição de aberturas, aspectos fundamentais no projeto arquitetônico bioclimático. Entendemos que este trabalho representa uma contribuição para o ensino do conforto ambiental e do projeto arquitetônico e urbano.
\end{abstract}

Palavras-chave: Heliodon, controle numérico, concepção bioclimática, simulação física.

\begin{abstract}
The teaching of bioclimatic architectural design involves, among many things, the knowledge of solar geometry, a fundamental element for the environmental performance of the building. Currently, due to the user-friendliness of computer simulation programs, architecture students undertake insolation studies by using numerical models, which alienates the actual knowledge of solar geometry. This paper presents a physical simulation equipment, controlled by computer, which was developed for the Laboratory of
\end{abstract}


Environmental Comfort and Sustainability - ECOS, and its potential application in education. It is the result of an applied research aimed to develop an innovative version - numeric controlled - of a classical device - the Heliodon. The objective of the article is to describe the materials and methods used in the development of the equipment and to present a pilot test conducted for validation of the device, based on comparative analysis of physical and computational simulation. The research methodology was based on the literature review of other similar equipment, incorporating as innovation, the numerical control. The results of the pilot test showed the suitability of the device in relation to the overall functioning and solar angles, however, there is a need of adjustment on lighting projector due to the deformation of the angles of light incidence. The tests demonstrate that using the Heliodon, together with computer simulations, students have a more complete instrumental to understand the physical phenomenon of the apparent path of the sun. This knowledge improves the expertise on building orientation and arrangement of windows, fundamental aspects of bioclimatic architectural design. We believe that this work represents a contribution to the teaching of environmental comfort and to the architectural and urban design.

Keywords: Heliodon, numerical control, bioclimatic design, physics simulation.

\section{INTRODUÇÃO}

No entendimento de que o conhecimento do fenômeno físico da trajetória aparente do sol é fundamental para a realização de projetos com qualidade ambiental, o curso de Arquitetura e Urbanismo da UFJF investe nos métodos físicos para o ensino do conforto ambiental. Entre eles, a simulação experimental através do heliodon permite apoiar a compreensão da geometria solar e testar alternativas para o projeto da edificação.

O heliodon é um equipamento de simulação experimental da trajetória solar, cujo objetivo é auxiliar o ensino da concepção arquitetônica bioclimática, através de análises da incidência solar em maquetes de edificações ou de espaços urbanos. As vantagens dos estudos com a utilização do heliodon quando comparado com análise por cartas solares ou programas computacionais são a visualização imediata de áreas iluminadas e sombreadas de ambientes nas diversas horas do dia e épocas do ano, e a possibilidade de manipulação rápida do objeto analisado para estudos de implantação, orientação de aberturas, dispositivos de proteção.

Os equipamentos existentes apresentam alguma dificuldade de manuseio para o posicionamento dos ângulos de azimute e altura solar. Justificou-se portanto o desenvolvimento do projeto de um heliodon que fosse capaz de permitir controle automatizado para facilitar seu uso no ensino de arquitetura.

Este artigo trata da concepção, construção e aplicação de um heliodon computadorizado, para ferramental didático do Laboratório de Conforto Ambiental e Sustentabilidade - ECOS do curso de Arquitetura e Urbanismo da Universidade Federal de Juiz de Fora (UFJF). O objetivo deste trabalho é apresentar as características da construção desta versão inovadora de heliodon, comandado através de interface computadorizada e apresentar o estudo de caso piloto de aplicação em estudo de proteção solar para edificações da UFJF. Buscou-se ainda testar o equipamento através de comparação de resultados entre a simulação com maquete física no heliodon e a simulação com maquete eletrônica.

A primeira parte do artigo apresenta as características físicas, construtivas e funcionais do equipamento. A segunda parte apresenta uma discussão sobre a adoção do equipamento no ensino de conforto ambiental e do projeto arquitetônico bioclimático.

O projeto de pesquisa para concepção e construção do heliodon foi financiado pela FAPEMIG, envolvendo professores dos cursos de Arquitetura e Urbanismo, Engenharia de Produção, Energia Elétrica e Engenharia Civil, cinco bolsistas de Iniciação Científica, estudantes do Programa de Educação Tutorial da Engenharia Elétrica e duas bolsistas de Iniciação Científica Jr do Ensino Médio. 


\section{FUNDAMENTAÇÃO}

A simulação do projeto arquitetônico pode ser realizada através de modelos físicos ou de modelos computacionais. A simulação experimental através de maquetes e simuladores físicos permite uma fácil manipulação do modelo arquitetônico em estudo, criando oportunidades de testar alternativas de orientação da edificação, variações em aberturas e proteções, com possibilidades de respostas visuais da aparência final do projeto, de forma a se verificar qualitativamente as melhores alternativas a serem desenvolvidas. Destaca-se que o estudo dos horários de insolação e ângulos que os raios solares incidem nas superfícies da edificação, bem como o estudo das aberturas para iluminação e ventilação são indispensáveis para o conforto ambiental de uma edificação. Estudos através de modelos reduzidos da edificação em heliodon podem determinar as épocas do ano, horários e ângulos de incidência com que a radiação solar chega a um ponto dado do espaço, para com isto, conceber o projeto de forma a melhor explorar orientações, aberturas e dispositivos de proteção (RIVERO, 1986).

Os instrumentos utilizados para o estudo das trajetórias solares são bastante variados. Vão desde ferramentas simplificadas como os diagramas solares e relógios de sol; passando por simulação em modelos físicos até simulações computacionais de diversos níveis de complexidade (CORBELLA, 2003).

Entre os principais equipamentos indicados para este tipo de aplicação está o heliodon, um equipamento simulador do movimento aparente do sol utilizados nos estudos de orientação solar e projeção de sombras nos edifícios ou no recinto urbano, através de maquetes ou modelos reduzidos. Visa facilitar o estudo de ambientes complexos, onde a aplicação de técnicas de projeção geométrica consumiria muito tempo. Por sua facilidade de operação e resposta imediata contribui para objetivos didáticos de tornar mais facilmente acessível a visualização da geometria solar, sendo, largamente utilizado no ensino da arquitetura e urbanismo. Permite a visualização imediata das áreas iluminadas e sombreadas do modelo, auxiliando no estudo de como a edificação se comportará em relação à incidência de luz solar em várias datas e horas do dia (LABCON-UFMG, 2010).

O heliodon projetado visa facilitar o posicionamento de ângulos de azimute e altura solar, o que representa, em geral, a maior dificuldade no manuseio deste tipo de equipamento. Para tal, uma interface para entrada de dados de dias e horas e um sistema de comando permitem transferir os dados do computador ao equipamento, fazendo com que ele gire para a posição de azimute e altura solar correspondente ao momento que se deseja analisar.

\section{MATERIAS E MÉTODOS}

\subsection{Características físicas do heliodon}

O projeto do heliodon desenvolvido no Laboratório ECOS (Figura 1) foi concebido a partir da pesquisa de modelos que foram desenvolvidos por outros laboratórios de conforto. O equipamento projetado visou, como diferencial, a movimentação por meio de comando numérico para posicionamento do refletor que simula o sol no dia e horário desejados (ZAMBRANO, CASTRO, 2010). O equipamento compreende uma mesa em MDF revestida com laminado melamínico, com tampo circular, tendo uma borda externa giratória (giro em torno do eixo vertical da mesa) e a parte central fixa, para apoio da maquete a ser estudada. Uma haste móvel (giro em torno do eixo horizontal da mesa) em perfil de alumínio (com sistema de contra pesos) sustenta um refletor parabólico que simula a luz solar. O giro da borda do tampo da mesa permite orientar o 
azimute e a movimentação da haste que suporta o refletor permite posicionar a altura solar. O tampo da mesa foi projetado com dimensão de $2,20 \mathrm{~m}$ de diâmetro e a haste com raio de $1,30 \mathrm{~m}$, o que permite a simulação com maquetes com dimensões máximas aproximadas de $0,80 \mathrm{~m} \times 0,80 \mathrm{~m} \times 0,80 \mathrm{~m}$.

\section{Figura 1 - Modelo tridimensional do projeto do heliodon}

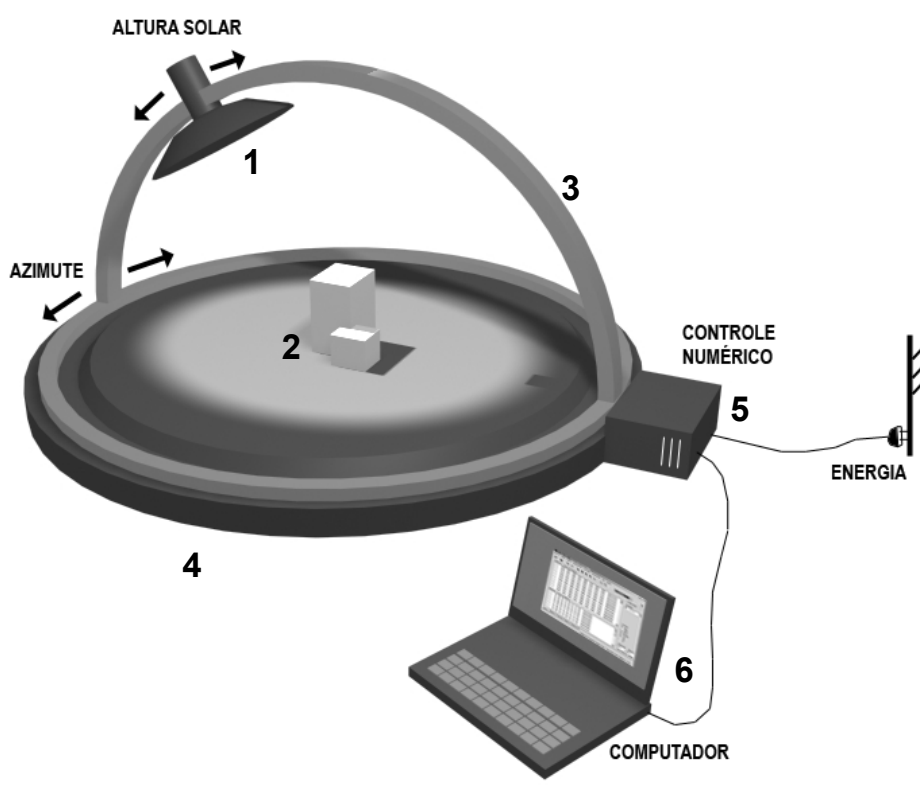

Legenda:

1 - Refletor parabólico com lâmpada halógena bipino de 150w simulando a posição do sol nos diversos dias e horas do ano;

2 - maquetes a serem analisadas em heliodon;

3 - haste móvel controlada por comando numérico para ajuste da altura solar;

4 - Borda do tampo giratória controlada por comando numérico para ajuste do azimute;

5- Hardware de controle numérico;

6- Programa de interface do usuário para entrada do dia e

Fonte: ZAMBRANO, CASTRO, 2010. horário a ser simulado.

Um software de interface do usuário permite a inserção da latitude do local bem como dos dias e horas que se deseja simular. Este software faz a tradução dos dados inseridos para azimutes e alturas solares e promove a movimentação da haste do equipamento para a posição correspondente. $O$ equipamento permite ainda uma visualização continuada ao longo do dia, das diversas posições do sol.

Figura 2- Vista frontal do heliodon

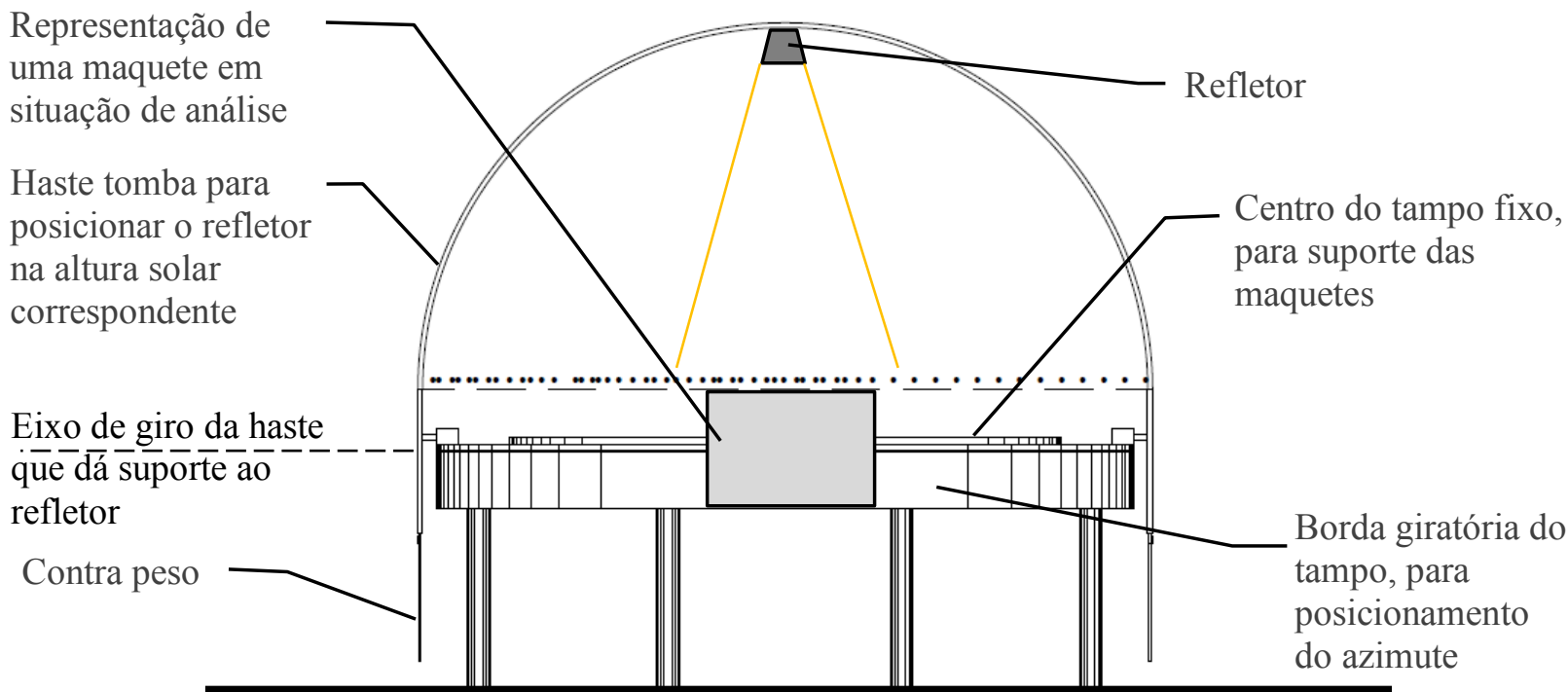

Fonte: os autores. 
Figura 3- Seção do heliodon onde se observa a borda giratória do tampo e a haste móvel

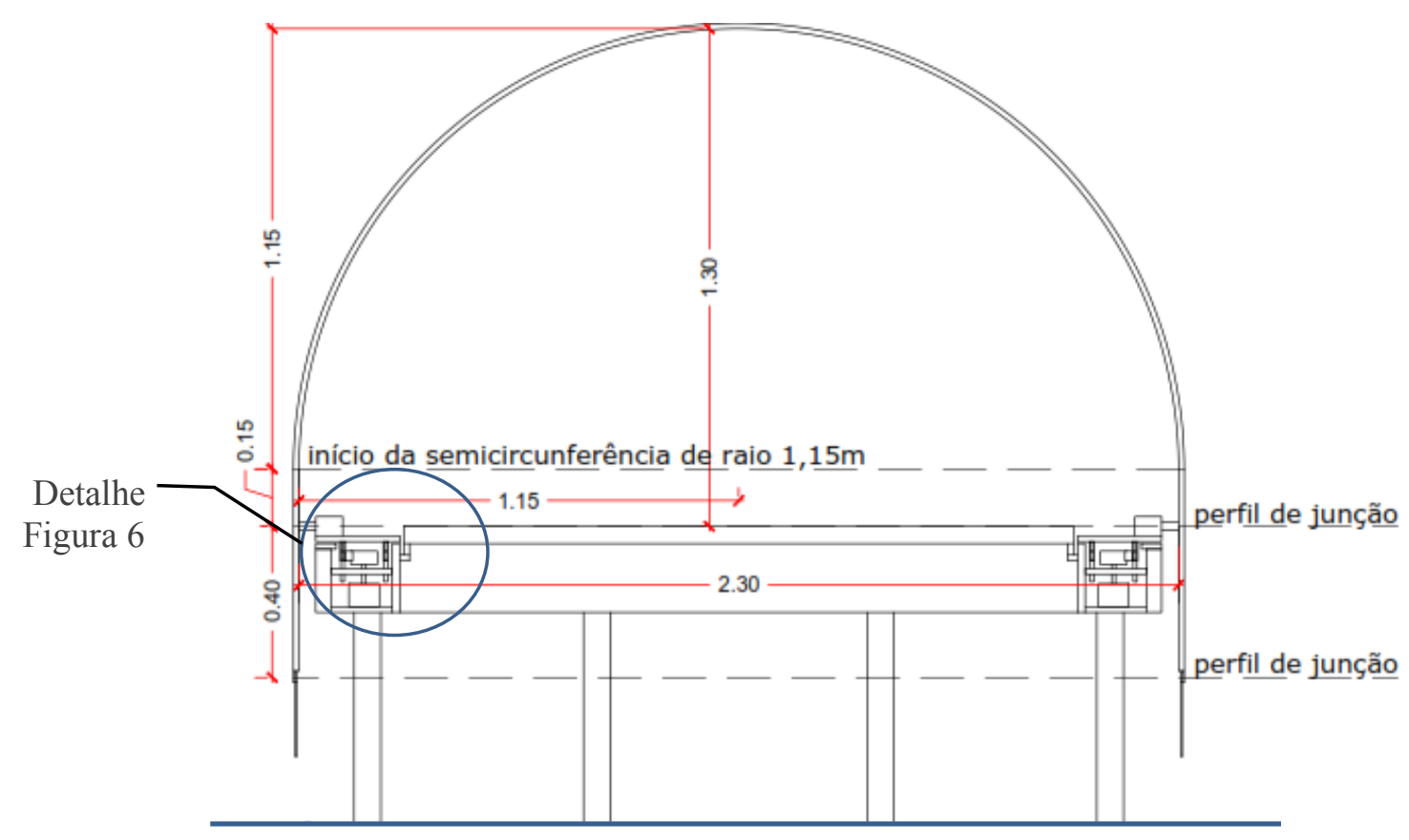

Fonte: os autores.

\section{Figura 4 - Secção da borda do tampo giratório - Detalhe do sistema de cremalheiras e conexão com o motor}

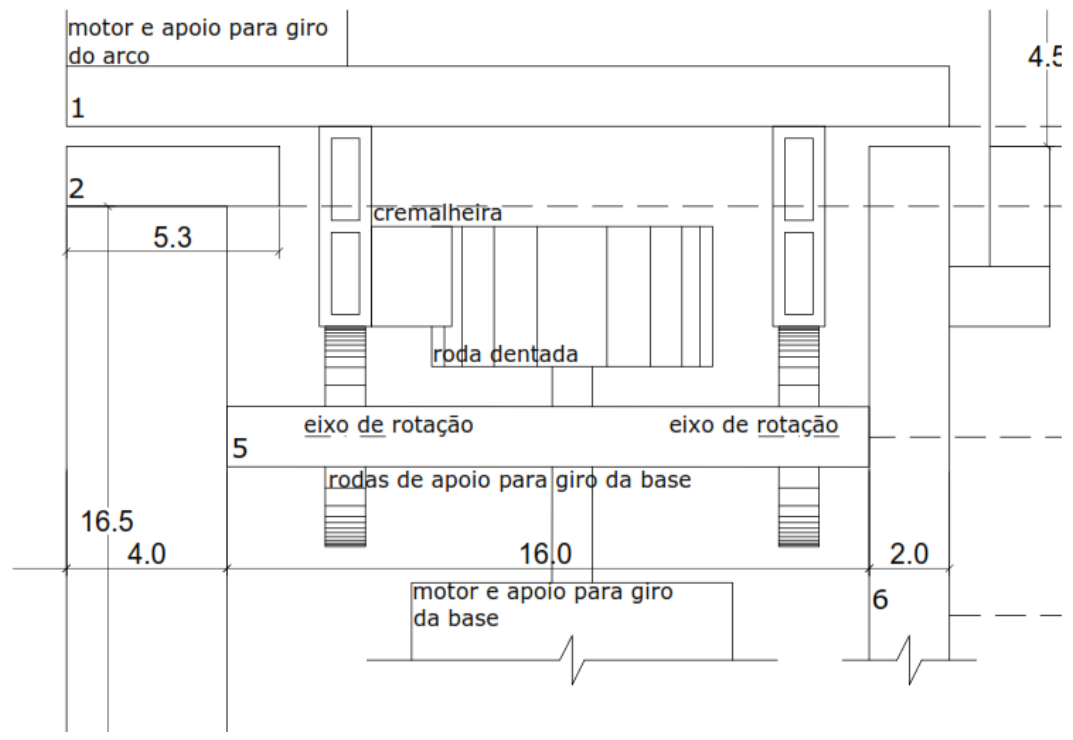

Fonte: os autores.

A imagem a seguir apresenta o heliodon já finalizado, evidenciando a haste em alumínio e o refletor em funcionamento (figura 5). $\mathrm{O}$ detalhe da parte central (figura 6) mostra a marcação em adesivo dos ângulos de azimute (ZAMBRANO, CASTRO, 2013). 
Figura 5 - Heliodon finalizado

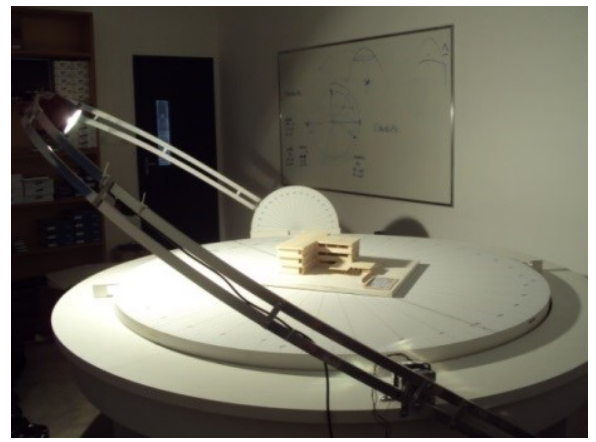

Figura 6 - Detalhe da parte fixa do tampo

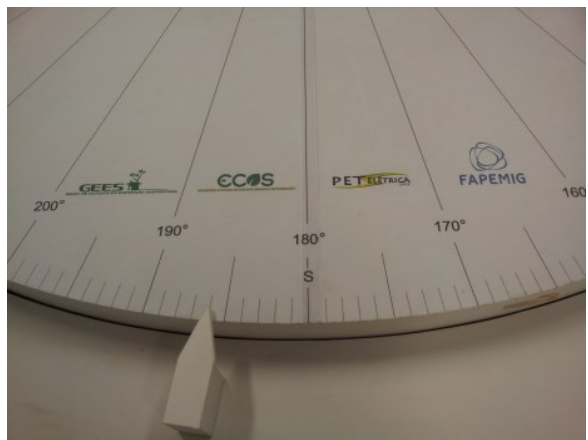

\subsection{Automação do equipamento}

\subsubsection{Funcionamento}

Para realizar a movimentação do heliodon foi necessário desenvolver um dispositivo capaz de traduzir as informações inseridas através da interface em sinais de acionamento para os motores. Com esta finalidade, foi escolhido o microcontrolador Arduino (ARDUINO, 2014) a fim de fazer a leitura e acionamento dos drivers. Esta escolha baseou-se no seu baixo custo, fácil manuseio e programação, além de ser uma plataforma FOSS - Free and Open Source Software (ORACLE TECHNOLOGY NETWORK, s/d), livre e aberta.

Para a automação do processo foi utilizado uma estrutura em malha aberta (OGATA, 2003), selecionada levando-se em consideração a alta precisão dos motores de passo e drivers escolhidos.

\subsubsection{Interface}

Foi desenvolvida uma interface capaz de tratar os dados de entrada que o usuário deve fornecer e, a partir destes valores, calcular a posição adequada da mesa. Como mostrado na figura a seguir, o usuário deve inserir os valores de latitude, longitude, data e horário do local em análise. A partir destes valores, o programa calcula o azimute e a inclinação correspondentes e apresenta a carta solar do determinado dia. A carta solar é um mapa que mostra as posições do Sol durante um dia na localização selecionada. Note que o horário escolhido aparece destacado no diagrama da figura abaixo, a fim de mostrar para o usuário a posição definida para o heliodon.

Figura 7 - Interface desenvolvida

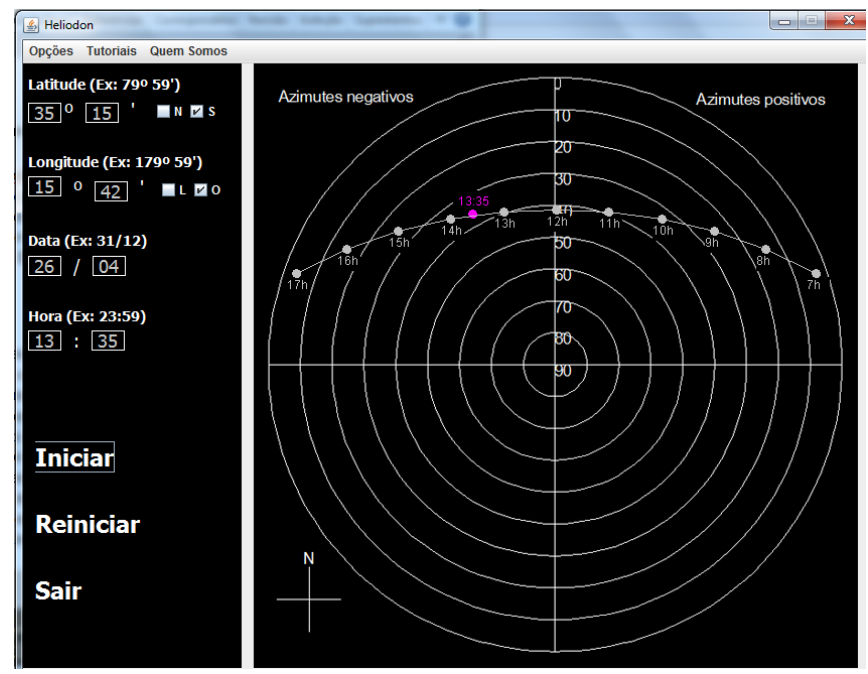


A interface apresenta ainda as opções de "Quem Somos" e "Tutoriais". Estes itens de menu são apenas informativos e respectivamente fazem referência aos criadores do projeto e a algumas informações úteis ao usuário que estiver com dificuldade de utilizar o programa. Vale salientar, que a interface foi programada em Java (ORACLE TECHNOLOGY NETWORK, s/d), utilizando o programa NetBeans IDE.

\subsubsection{Calibragem do heliodon}

Tratando-se de um sistema de malha aberta, ou seja, que não utiliza sensores para controle de posicionamento do heliodon, foi necessário desenvolver uma opção de calibragem do instrumento. Nesta opção o usuário ajusta manualmente o módulo e insere a posição no programa. Com esta calibragem o heliodon se posiciona automaticamente para as próximas posições, porém não há um dispositivo para reduzir os erros decorrentes do uso, logo torna-se necessário o ajuste manual quando o módulo perde precisão.

\subsubsection{Circuito completo}

Para realizar o gerenciamento do processo foi necessário desenvolver um painel a fim de realizar o controle e alimentar os motores necessários para o posicionamento da mesa. Na figura 9 pode ser visto o circuito completo para ser utilizado no heliodon. Dentre eles está a fonte de $68 \mathrm{~V}$ para alimentação dos drivers (componentes que realizam a comunicação dos dados entre o arduino e os motores). $\mathrm{O}$ arduino é o componente que permite o acionamento dos motores e é conectado a um computador, de onde é realizado o posicionamento automático.

Figura 8 - Função calibragem

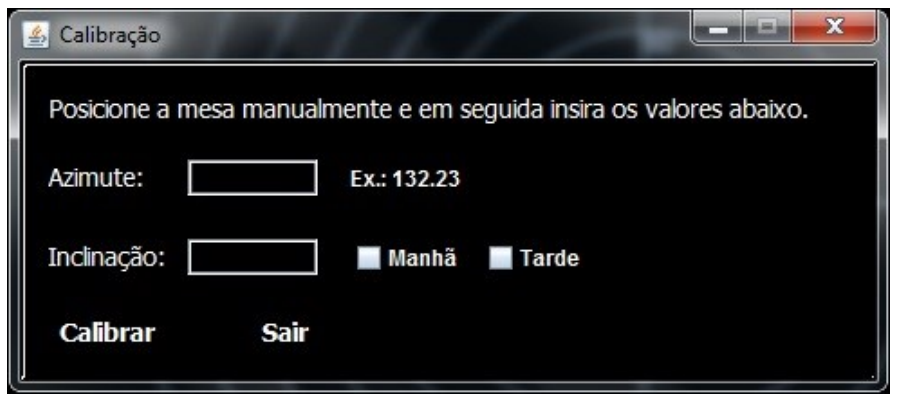

Figura 9 - Circuito completo

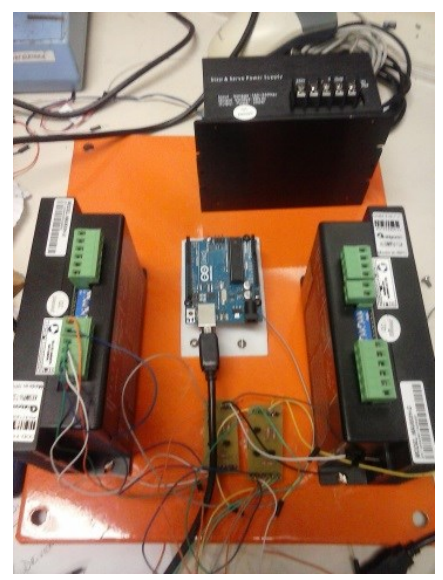

\subsubsection{Circuito de proteção}

A alimentação dos motores é realizada através de fios inseridos dentro da estrutura física da borda giratória do equipamento, tendo sido necessário desenvolver uma estrutura de proteção para garantir que o movimento do motor não ultrapasse o limite físico requerido. Para tal, foram utilizadas duas chaves fim-de-curso que limitam o movimento do motor responsável pelo azimute.

Outro recurso para garantir a segurança dos equipamentos e do usuário foi a inclusão de uma chave de emergência colocada junto ao painel desenvolvido para o acionamento. Trata-se de uma chave cogumelo e pode ser facilmente acionada pelo usuário em caso de mau funcionamento do modelo. Ao ser acionada a chave corta automaticamente a alimentação do circuito. 


\section{TESTES DO HELIODON}

Com o objetivo de testar o instrumento, foram escolhidas algumas edificações da UFJF para serem analisadas. Optou-se por selecionar cinco edifícios de usos distintos, de forma a abordar problemáticas de insolação presentes nas variadas tipologias arquitetônicas

Em paralelo aos ensaios no heliodon, foram realizados também estudos com maquetes eletrônicas no software SketchUp (TRIMBLE, 2013) como forma de comparação de resultados. Os modelos eletrônicos foram executados com o mesmo nível de detalhamento dos modelos físicos, de forma a evitar erros por incompatibilidade dos modelos.

Apresentamos a seguir uma pequena parte dos estudos realizados a fim de ilustrar os procedimentos adotados para os testes, os resultados obtidos e exemplificar suas possibilidades no ensino do conforto ambiental e do projeto arquitetônico.

\subsection{Simulações e Análises}

Para as simulações e análises selecionou-se dia e horários em que a presença da insolação direta dentro das edificações seria indesejada. Foi escolhido o dia 22 de dezembro, que por se tratar do solstício de verão no hemisfério sul, pode apresentar maior potencial de desconforto térmico, caso as fachadas e aberturas estejam muito expostas ou desprotegidas da radiação solar direta. As maquetes foram posicionadas no centro do heliodon orientadas em relação ao norte geográfico e o equipamento ajustado para azimute e altura solar correspondentes ao dia 22 de dezembro, sendo simulados dois horários ( 8 horas e 17 horas).

O mesmo procedimento foi repetido na modelagem em SketchUp, com orientação da edificação, inserção de dados de latitude $\left(21^{\circ} 45^{\prime} 51^{\prime}\right.$ 'S $)$ e longitude $\left(43^{\circ} 21^{\prime} 01^{\prime \prime} \mathrm{W}\right)$ da cidade de Juiz de Fora e verificação da incidência solar no dia e horários planejados.

\subsection{Simulação da Edificação do Curso de Arquitetura e Urbanismo}

Apresenta-se, como exemplo, a simulação da edificação do Curso de Arquitetura e Urbanismo. Inicialmente foram identificadas as fachadas críticas (NE e SO), onde a incidência da luz solar penetra mais profundamente pelas aberturas das grandes janelas presentes na edificação.

A simulação computacional realizada com maquete eletrônica no programa SketchUp permite verificar a compatibilidade dos resultados angulares de altura solar e azimute entre os dois tipos de simulação, porém com variação em relação aos ângulos das sombras projetadas na simulação no heliodon. As figuras a seguir apresentam os resultados das simulações tendo como alvo de estudo a fachada SO, que recebe o sol das 17 horas no solstício de verão.

\section{Figura 10 - Simulação heliodon}

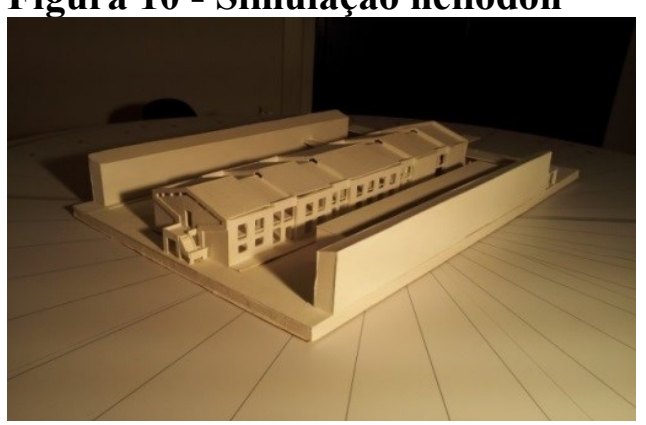

Figura 11 - Simulação computacional

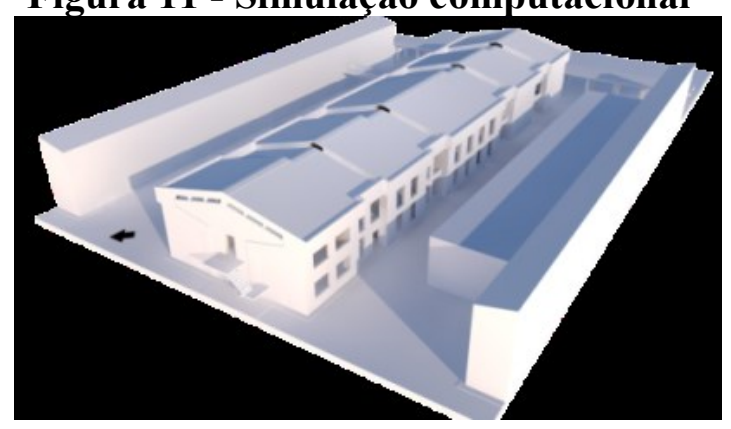


No processo identificou-se que a escala de confecção das maquetes das edificações era insuficiente para estudos mais detalhados de proteções solares a serem projetadas como soluções aos problemas identificados. Assim, após estudos mais gerais da edificação, para cada maquete simulada foi realizada uma maquete em escala ampliada de um trecho da fachada crítica, de forma a se poder simular a inserção de elemento de proteção que possibilitasse impedir a incidência solar direta nas aberturas. Estas maquetes foram submetidas à nova simulação.

\section{Figura 12 - Trecho da fachada com abertura sem proteção da entrada da luz solar direta.}

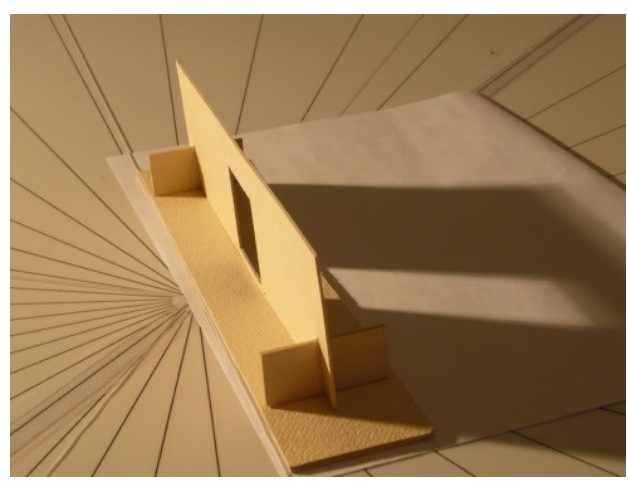

Figura 13 - Trecho da fachada com inserção do brise soleil horizontal regulável para proteção da abertura.

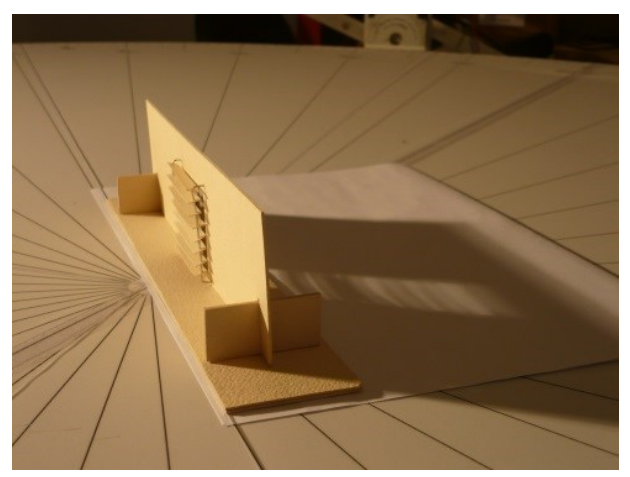

\section{CONCLUSÕES E CONSIDERAÇÕES FINAIS}

Os testes comparativos realizados entre simulações computacionais e simulações no heliodon validam o equipamento desenvolvido em termos de ângulos de azimute e altura solar a partir de uma latitude, dia e hora simulados. Entretanto, a adoção de luminária para simulação da luz solar, a uma distância próxima ao objeto analisado, mesmo sendo esta um refletor parabólico, gera um cone de iluminação que provoca distorções em relação ao que seria a luz solar verdadeira, que incide na forma de raios paralelos. Este fato pode ser observado nas comparações realizadas entre a simulação física e a simulação em maquete eletrônica no SketchUp, onde a iluminação é gerada a partir de raios paralelos, como acontece com a luz solar. A partir da identificação do problema, outros tipos lâmpadas e refletores estão sendo pesquisados com vistas as minimizar esta distorção. Indica-se ainda que o tamanho das maquetes a serem realizadas no heliodon seja inferior às dimensões máximas inicialmente previstas no projeto do equipamento $(0,80 \mathrm{~m} \times 0,80 \mathrm{~m} \times 0,80 \mathrm{~m})$.

A produção do heliodon fornece aos estudantes, ao Laboratório de conforto Ambiental e Sutentabilidade - ECOS e ao Curso de Arquitetura e Urbanismo um importante instrumento didático de auxílio à compreensão dos efeitos da trajetória solar sobre a edificação. Este pode ser utilizado em estudos laboratoriais das disciplinas de Conforto Ambiental, em análises nas disciplinas de Projeto de Arquitetura e Urbanismo ou em qualquer outra disciplina que objetive analisar a insolação sobre ambientes urbanos ou edificações. O processo de projeto e de produção do heliodon proporcionou o desenvolvimento de conhecimento para todos os envolvidos dos cursos de Arquitetura e Urbanismo, Elétrica, Mecatrônica e Automação. Seu uso permitirá a multiplicação deste conhecimento para outros estudantes que queiram, através de seu entendimento e manuseio, aprofundar seus conhecimentos, tanto no estudo do funcionamento do equipamento, como na sua aplicação em simulação de ambientes.

Os testes realizados nos estudos de caso geraram informações para adequações do 
funcionamento dos mecanismos do heliodon e na forma de seu uso final, com maior segurança e precisão.

\section{AGRADECIMENTOS}

A todos os parceiros deste projeto, sem os quais o desenvolvimento deste modelo de heliodon não teria sido possível. A FAPEMIG pelo apoio recebido para desenvolvimento do projeto de pesquisa para desenvolvimento do heliodon e para a apresentação deste trabalho. A Universidade Federal de Juiz de Fora e Faculdade de Engenharia pelo apoio para a adequação das instalações físicas do Laboratório ECOS para a instalação do heliodon e para a apresentação deste trabalho.

\section{REFERÊNCIAS}

ARDUINO CC. Arduino web site. Copyright Arduino, 2014. Disponível em: $<$ http://www.arduino.cc/> Acesso em: 11 de Maio de 2014.

CORBELlA, O; YANNAS, S. Em busca de uma Arquitetura Sustentável para os trópicos. Rio de Janeiro: Revan. 2003

LABCON-UFMG. Laboratório de Conforto Ambiental e Eficiência Energética em Edificações. Equipamentos. Website do LABCON-UFMG. Disponível em: http://www.arquitetura.ufmg.br/labcon/?pag=texto\&id=41. AcessO em março de 2010.

OGATA, K. Engenharia de Controle Moderno. $4^{\text {a }}$ Edição. Minas Gerais: PrenticeHall (Pearson), 2003.

ORACLE TECHNOLOGY NETWORK. Java Web site. s/d. Disponível em: $<$ http://www.java.com/pt_BR/> Acesso em: 11 de Maio de 2014.

RIVERO, Roberto. Arquitetura e Clima: Acondicionamento Térmico Natural. Porto Alegre: Luzzato Editores. 1986

TRIMBLE. SketchUp. A maneira mais fácil de desenhar em 3D. Pagina internet. Copyrights Trimble Navegação Limitada, 2013. Disponível em: http://www.sketchup.com/pt-BR. Acessado em 09 de maio de 2014.

ZAMBRANO, L.M.A., CASTRO, E.B. Estudo de Dispositivos de Proteção para o Conforto Térmico em Edificações da UFJF Baseado na Aplicação de Simulação Experimental em Heliodon. Projeto de Pesquisa para o edital FAPEMIG 01/2010. Juiz de Fora, 2010.

ZAMBRANO, L.M.A., CASTRO, E.B. Relatório Final - TEC APQ 01935/10. Estudos relacionados ao Conforto Ambiental de edificações da UFJF baseados na aplicação de simulação experimental em heliodon. Juiz de Fora, 2013. 